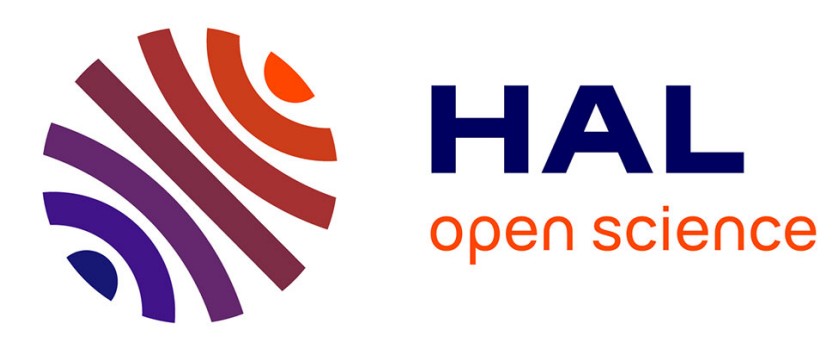

\title{
Surface plasmon routing along right angle bent metal strips
}

\author{
J. C. Weeber, M. U. Gonzalez, Anne Laure Baudrion, Alain Dereux
}

\section{To cite this version:}

J. C. Weeber, M. U. Gonzalez, Anne Laure Baudrion, Alain Dereux. Surface plasmon routing along right angle bent metal strips. Applied Physics Letters, 2005, 87 (22), pp.221101. hal-00472596

\section{HAL Id: hal-00472596 https://hal.science/hal-00472596}

Submitted on 12 Apr 2010

HAL is a multi-disciplinary open access archive for the deposit and dissemination of scientific research documents, whether they are published or not. The documents may come from teaching and research institutions in France or abroad, or from public or private research centers.
L'archive ouverte pluridisciplinaire HAL, est destinée au dépôt et à la diffusion de documents scientifiques de niveau recherche, publiés ou non, émanant des établissements d'enseignement et de recherche français ou étrangers, des laboratoires publics ou privés. 


\section{Surface plasmon routing along right angle bent metal strips}

J-C Weeber, M. U. González, A-L Baudrion and A. Dereux

Laboratoire de Physique de l'Université de Bourgogne, UMR CNRS 5027,

9 avenue A. Savary, BP 47870, F-21078 Dijon, France

(July 5, 2005)

An efficient routing of surface plasmon polaritons (SPP) is of fundamental importance in the development of SPP-based photonics. This paper demonstrates the feasibility of routing SPP along metal stripes waveguides with $90^{\circ}$ bents by the integration of tilted micro-gratings acting as Bragg mirrors in the strips. The quantitative characterization of the mirrors efficiency, performed by means of photon scanning tunneling microscopy, shows that losses as low as $1.9 \mathrm{~dB}$ can be achieved. Moreover, SPP 50/50 beamsplitters have been obtained by an appropriate design of the Bragg mirrors constituting elements. 
Surface plasmon polaritons (SPP) propagating on finite widths metallic thin films have recently received much interest in the context of the trends towards the development of optical devices featuring reduced sizes. When encapsulated into a dielectric matrix, thin metal strips (MS) can sustain long-range surface plasmon polariton (LR-SPP) modes [1] with typical attenuation of a few $\mathrm{dB} / \mathrm{mm}$ at telecommunication frequencies. Passive or even active LR-SPP based devices such as splitters or modulators $[2,3]$ have been very recently demonstrated. If the metal stripes are deposited on a substrate with a refractive index different than that of the superstrate, the electromagnetic field of the MS-SPP modes penetrates more deeply into the metal, leading to propagation distances much smaller than in the symmetric case $[4,5]$ but providing a higher vertical confinement. Asymmetric MS waveguides are therefore promising for applications where the required propagation distances are short, such as downsizing of optical components [6] or SPP sensors.

In practical contexts, guiding SPP along bent MS paths will be required. Unfortunately, MS are weakly guiding systems [7] and, therefore, large scattering losses are expected for SPP propagating along sharply bent MS. On the other hand, SPP Bragg mirrors comprised of lines of closely packed gold nanoparticles were found to be efficient for deflecting a SPP traveling along an extended thin film [8] and micro-gratings of slits engraved in MS have been used to achieve the back-reflection of SPP modes sustained by the MS [9]. However, as alternative to the lossy bent MS paths, the performance of tilted SPP Bragg mirrors in achieving SPP routing in MS branches has not been reported so far. In this work, we have developed Bragg mirrors able to guide MS-SPP modes along $90^{\circ}$ sharp bents, and their efficiency has been determined by means of a near-field optical microscope. Bent losses as low as $1.9 \mathrm{~dB}$ are obtained using mirrors comprised of gratings of gold ridges. We also show that MS-SPP modes splitters can be obtained by adjusting the number of ridges in the mirrors.

A SPP is Bragg reflected when encountering a grating at an angle $\theta$ with respect to the direction of propagation if its in-plane wave vector $k_{s p}$ is given by:

$$
k_{s p}=\frac{k_{g}}{2 \cos \theta},
$$

where $k_{g}$ denotes the modulus of the grating reciprocal vector. Since a MS waveguide tightly confines the SPP field in the transverse direction [10], a grating with a given period can not establish the Bragg condition for all components of the field of the plane-waves expansion of a MS-SPP mode. In fact, instead of a single in-plane propagation direction (as it is the case with the collimated finite size SPP beams used in ref. [8], for example), a distribution of directions centered on the long axis of the MS is associated to a laterally confined MS mode. The reflection power of a given Bragg mirror is then expected to depend on the MS-SPP mode itself.
In order to assess the spreading of the SPP modes sustained by the reference $2.7 \mu \mathrm{m}$-wide MS used in the following, the sample shown in Fig. 1(a) has been fabricated by electron-beam lithography. It consists of a $60 \mathrm{~nm}$ thick gold film deposited on a ITO-doped glass substrate and featuring a MS connected to two larger areas. At the surface of the upper larger area, the SPP are excited at the gold/air interface in the Kretschmann-Raether configuration using a focused spot (wavelength in vacuum $\lambda_{0}=800 \mathrm{~nm}$ throughout this paper). The SPP launched on the extended thin film propagates from the top to the bottom of the image and couples to the SPP modes of the MS. Fig. 1(b) shows the photon scanning tunneling microscope (PSTM) [11] image recorded over the area corresponding to the white dashed perimeter displayed on Fig. 1(a). In agreement with previous studies [10,9], the near-field image of the MS exhibits a characteristic electric field intensity distribution attributed to the modal distribution of the MS-SPP $[12,10]$. When arriving to the lower wider area $(8 \mu \mathrm{m}$-width $)$, the modal structure vanishes and the intensity distribution of MS-SPP spreads out. The scattering spot at the bottom edge of the wide thin film area allows to determine the spreading angle $\phi 17 \pm 2^{\circ}$ (see Fig. 1(b)). This spreading imposes an important constraint to the subsequent placement of the mirror regarding to the incident MS in order to minimize the reflectivity losses that it would induce. The mirror should be placed at the very end of the "input" MS where the light is coming from to be routed to an "output" MS, bent at the desired angle ( $90^{\circ}$ in this work).

Figure 2(a) shows the configuration designed to measure the reflection efficiency of the Bragg mirrors integrated into MS waveguides. The samples include a reference straight stripe and a $90^{\circ}$ bent strip equipped with a tilted Bragg mirror. They are fabricated by using a twosteps electron beam lithography process: the Bragg mirrors, consisting of $60 \mathrm{~nm}$ high gold ridges with a period $d$ of $557 \mathrm{~nm}$, were fabricated first while the MS were exposed during the second step after an appropriate alignment procedure. With the aim of assessing the integrated Bragg mirrors reflectivity, we have compared the PSTM images of both reference and bent strips. The methodology of the reflectivity measurement relies on the comparison of the scattering spot intensities at the output ends of both kinds of waveguides, normalized by their corresponding input levels. Owing to the propagation damping of the MS-SPP modes, this comparison makes sense only if the length $L_{t}$ ot of the reference strip is equal to the total length $L_{1}+L_{2}$ of the bent MS. Figs. 2(b) and (c) show typical PSTM images of the reference and bent MS. Denoting the maximum scattering spot intensities at the output end of the reference and bent MS $I_{\text {out }}^{\text {ref }}$ and $I_{\text {out }}$ respectively, the losses related to the $90^{\circ}$ deflection of the MS-SPP modes are given by:

$$
\mathcal{L}[\mathrm{dB}]=-10 \log \frac{I_{\text {out }} I_{\text {in }}^{\text {ref }}}{I_{\text {out }}^{\text {ref }} I_{\text {in }}}
$$


where $I_{i n}^{r e f}$ and $I_{i n}$ are respectively the maximum nearfield intensities detected over the input strip of the reference and bent MS. The solid line white arrows included in Figs. 2 (b) and (c) point to the areas where these intensities are measured.

By performing smaller PSTM scans of the regions of interest (not shown), the near-field and scattering intensities involved in Eq. 2 have been measured accurately and bent losses of $\mathcal{L}=2.8 \mathrm{~dB}$ have been found for the bent MS shown in Fig. 2(a). This technique for the computation of the losses assumes that the scattering process efficiency is the same at the output end of the bent and of the reference MS. However, the scattering of the SPP mode is expected to depend on the local geometry of the thin metal film at the end of the waveguide. In order to estimate the error introduced by the deviation of the scattering efficiency from one MS to another, PSTM images of up to six reference MS have been recorded using two different probes. On the basis of these images, we found a relative deviation for the ratio $R_{r e f}=\frac{I_{o u t}^{r e f}}{I_{\text {in }}^{r e f}}$ of about $\frac{\Delta R_{r e f}}{R_{\text {ref }}}=6 \%$. Assuming the same relative deviation for the ratio $\frac{I_{\text {out }}}{I_{\text {in }}}$, we end up with an absolute error $\Delta \mathcal{L}$ of $0.6 \mathrm{~dB}$.

Three main channels contribute to the losses computed according to Eq. 2: the transmission through the mirror, the scattering by the mirror and insertion losses of the reflected SPP mode into the output stripe of the bent waveguide. The two first losses channels being intrinsic to the mirror, the insertion losses can be nevertheless minimized by controlling the location of the output strip relative to the mirror. Indeed, on the PSTM image of Fig. 2(c), it can be observed a scattering spot (pointed out by the white dashed arrow) showing that part the reflected field has not been coupled within the output stripe SPP modes. Figure 3(a) shows a bent waveguide where the distance $s$ that takes into account the lateral position of the output stripe relative to the mirror is $s=1.6 \mu \mathrm{m}$ instead of $s=2.2 \mu \mathrm{m}$ as in the bent MS shown in the previous sample (Fig. 2). The corresponding PSTM image (Fig. 3(b)) does not exhibit anymore this misalignment scattering spot and accordingly smaller losses $(\mathcal{L}=1.9 \pm$ $0.6 \mathrm{~dB}$ ) are obtained. Similar losses values within the error range have been obtained for two equivalent samples. From these measurements, we can conclude that the MSSPP mode reflectivity of the $45^{\circ}$ tilted Bragg mirrors is at least $65 \%$, assuming zero insertion losses.

Such a high reflectivity may sound rather surprising when considering the previously observed large spreading of the MS modes. Indeed, for the plane waves of the MS mode spectrum traveling with the largest in-plane angle with respect to the longitudinal MS axis, the reflectivity of the Bragg mirror is expected to be poor because the mirror is designed to operate at an angle of incidence corresponding to the MS axis. However, these components being of small amplitude, the effect of their poor reflection should be just a slight broadening of the reflected beam compared to the incident one. Moreover, it has been shown that a sinusoidal grating can open a gap in the dispersion curve of plasmon polaritons propagating along directions within an angular range of about $\pm 10^{\circ}$ with respect to the angle of incidence matching the Bragg condition [13]. Even if the geometrical profiles of the mirrors we consider are far from sinusoidal (the interaction of the SPP can be significantly influenced by the geometrical profile of the grating [14]), this suggests that a Bragg mirror can be efficient for angles of incidence within a range of several degrees, thereby explaining qualitatively the large reflection of the MS-SPP modes.

Up to now, we have considered mirrors with a fixed number $N_{l}=10$ of ridges. In this last section, we use mirrors featurings lower $N_{l}$ to achieve MS-SPP modes splitters. Figure 4(a) shows the SEM image of one of the fabricated MS splitters. A Bragg mirror comprised of only three ridges $\left(N_{l}=3\right)$ has been integrated at the crossing of the two SPP waveguides. The output strips labeled in Fig. 4(a) as (1) and (2) are strictly equivalent in length and width. From the PSTM image of this device, shown in Fig. 4(b), it can be seen that the scattering spots at the output of each branch of the splitter are of same intensity. This intensity corresponds to $40 \pm 5 \%$ of the scattering spot intensity found at the end of a reference strip, as determined by the method described above. This result demonstrates the fabrication of a 50/50 MS-SPP mode splitter. By increasing to 5 the number of ridges in the Bragg mirror, a 70/30 MS-SPP modes splitter has been achieved (the intensities at the end of branch (2) and (1) have been found to be respectively $48 \pm 6 \%$ and $19 \pm 3 \%$ of that of a reference stripe).

In summary, we have performed quantitative near-field measurements of the reflectivity of Bragg mirrors integrated into SPP waveguides. We have found that,in spite of their lateral confinement, MS-SPP modes are efficiently routed along $90^{\circ}$ bents by using tilted Bragg mirrors provided that the lateral position of the mirrors relative to the input and output stripes is properly adjusted. Finally, we have demonstrated the fabrication of MS-SPP modes splitters opening the route towards the development of devices such as highly integrated SPP interferometers.

\section{ACKNOWLEDGMENTS}

This work was supported by the European Commission (Project NoE FP6-2002-IST-1-507879) and the regional council of Burgundy (ARCEN project).

[1] P. Berini, Phys. Rev. B 61, 10484 (2000). 
[2] S.I. Bozhevolnyi, T. Boltasseva, T. Sondergaard, T. Nikolajsen, K. Leosson, Opt. Commun. 250, 328 (2005).

[3] T. Nikolajsen, K. Leosson, S.I. Bozhevolnyi, Opt. Commun. 244, 345 (2004).

[4] P. Berini, Phys. Rev. B 63, 125417 (2001).

[5] B. Lamprecht, J. R. Krenn, G. Schider, H. Ditlblacher, M. Salerno, N. Felidj, A. Leitner, F. R. Aussenegg, J-C Weeber, Appl. Phys. Lett. 79, 51 (2001).

[6] A. Hohenau, J.R. Krenn, A.L. Stephanov, A. Drezet, H. Ditlblacher, B. Steiberger, A. Leitner, F.R. Aussenegg, Opt. Lett. 30, 893 (2005).

[7] R. Zia, A. Chandran, M. L. Brongersma, Opt. Lett. 30, 1473 (2005).

[8] H. Ditlbacher, J. R. Krenn, G. Schider, A. Leitner, F. R. Aussenegg, Appl. Phys. Lett. 81, 1762 (2002).

[9] J-C Weeber, Y. Lacroute, A. Dereux, E. Devaux, T. Ebbesen, C. Girard, M. U. González, A-L Baudrion, Phys. Rev. B 70, 235406 (2004).

[10] J-C Weeber, Y. Lacroute, A. Dereux, Phys. Rev. B 68, 115401 (2003)

[11] R. C. Reddick, R. J. Warmack, T. L. Ferrell, Phys. Rev. B 39, 767 (1989).

[12] R. Zia, M. D. Selker, M. L. Brongersma, Phys. Rev. B 71, 165431 (2005).

[13] W.L. Barnes, S.C. Kitson, T.W. Preist, J.R. Sambles, J. Opt. Soc. Am. A 14, 1654 (1997).

[14] M. Kretschmann, T.A. Leskova, A.A. Maradudin, Opt. Commun. 215, 205 (2003).
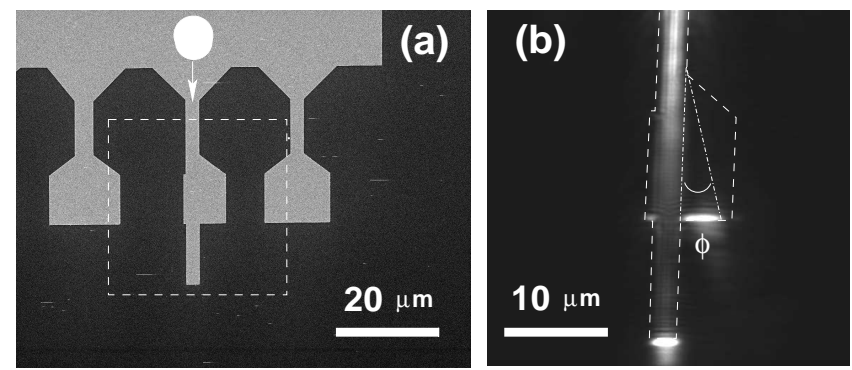

FIG. 1. (a) Scanning Electron Microscope (SEM) image of the sample used to study the spreading of the MS-SPP modes. The incident spot used to launch the incident SPP is located on the upper extended thin film area. (b) PSTM image corresponding to the white dashed perimeter on (a). The MS-SPP mode spreads out when it reaches the wider pad connected to the MS waveguide.

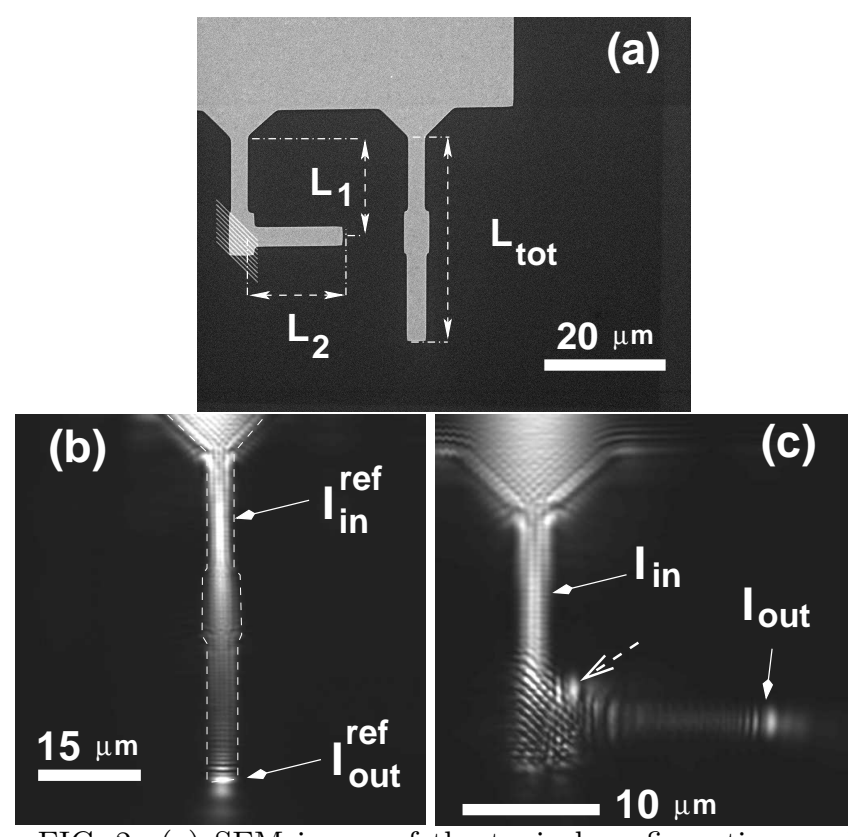

FIG. 2. (a) SEM image of the typical configuration used to measure the losses due to the guiding along a $90^{\circ}$ bent. The input and output stripes have a width of $2.7 \mu \mathrm{m}$ and $3.1 \mu \mathrm{m}$ respectively. For the bent MS, a Bragg mirror with a period of $557 \mathrm{~nm}$ and comprised of 10 gold ridges has been integrated at the crossing of the two strips. (b) (Resp. (c)) PSTM image of the straight (resp. bent) strip recorded for an incident wavelength of $\lambda_{0}=800 \mathrm{~nm}$.
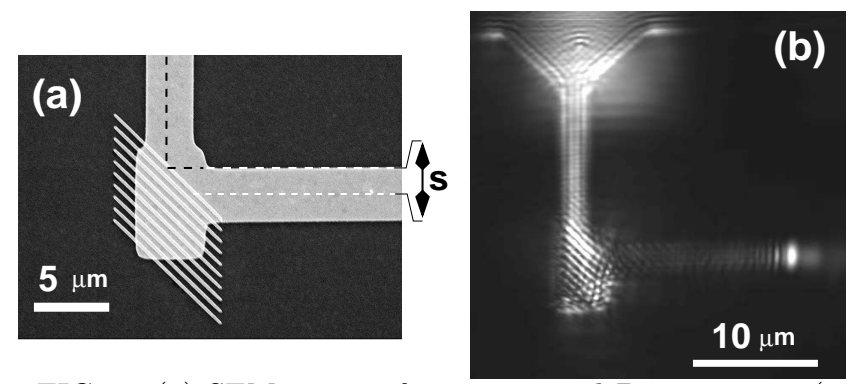

FIG. 3. (a) SEM image of an integrated Bragg mirror (10 lines, period $=557 \mathrm{~nm}$ ). The lateral shift of the mirror with respect to the output strip has been adjusted in order to minimize insertion losses. The distance $s$ between the center of the output strip and the line passing through the center of the first line of the mirror is equal to $1.6 \mu \mathrm{m}$. (b) Large scan PSTM image of the bent strip equipped with the mirror shown in (a). 


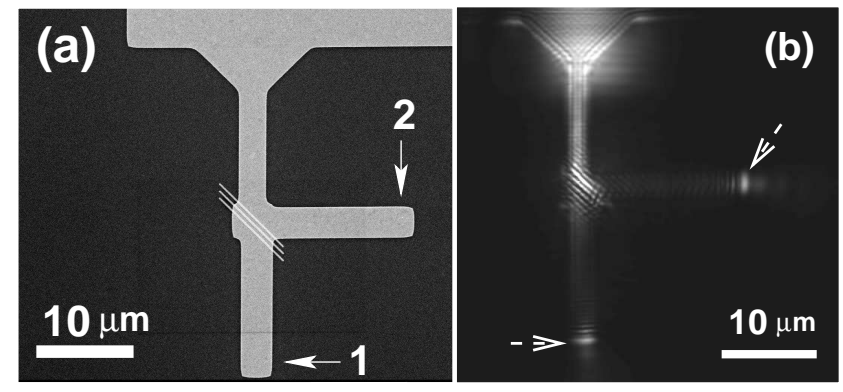

FIG. 4. (a) SEM image of a metal strip based 50/50 SPP splitter. A Bragg mirror with only three ridges is integrated at the crossing of the MS waveguides. (b) Large scan PSTM image of the splitter. The intensity of the scattering spot is the same at the end of both branches of the splitter. 